\title{
PRÁCTICA PROFESIONAL PENSADA Y NO PRODUCTO DE IMPROVISACIÓN. UNA MIRADA DESDE LA EDUCACIÓN A DISTANCIA
}

\author{
PROFESSIONAL PRACTICE
}

DESIGNED, AND NOT A PRODUCT

OF IMPROVISATION. A LOOK FROM

DISTANCE UNIVERSITY EDUCATION

\author{
Sandra Patricia Álvarez Pérez \\ Alba Lucia Díaz Delgadillo² \\ Universidad Pedagógica Experimental Libertador UPEL, Venezuela. \\ Escuela Tecnológica del Oriente, Bucaramanga - Colombia
}

\section{RESUMEN}

El presente artículo enfatiza la importancia de la práctica profesional de estudiantes universitarios de Uniminuto durante experiencias significativas en el sector empresarial. Las prácticas desde su carácter formativo buscan consolidarse como un proceso reflexivo y critico ayudando a comprender e interpretar la realidad de la profesión en áreas de conocimiento con

1 Sandra Patricia Alvarez Pérez, Especialista en Gerencia en Instituciones Educativas Universidad del Tolima, Candidata a Doctora en Educación Universidad Pedagógica Experimental Libertador UPEL, Administradora de Empresas Universidad Cooperativa de Colombia UCC, Instructora de Tecnología en Gestión Empresarial, Tecnología en Gestión Administrativa, Técnico en Asistencia Administrativa Servicio Nacional de Aprendizaje Sena, Red Administrativa y Financiera, Líder Semillero de Investigación Crisálida, Catedrática de Prácticas Profesionales y Administración de Empresas, Universidad Minuto de Dios UNIMINUTO, Facultad de Administración de Empresas, Empresaria, Asesora a estudiantes para tesis de grado, Universidad Minuto de Dios UNIMINUTO, Facultad Administración de Empresas. spatricialvarez@misena.edu.co. https://orcid.org/00000003-4952-5791.

2 Alba Lucia Díaz Delgadillo, Especialización en Pedagogía y didácticas Especificas Escuela Tecnológica del Oriente, Candidata a Doctora en Educación Universidad Pedagógica Experimental Libertador UPEL, Licenciada en Pedagogía Infantil Universidad del Tolima, Formada como Normalista superior, Docente Básica Primaria. dalbalucia@yahoo.es. https://orcid. org/0000-0003-2039-1582. 
aprendizajes significativos. Ellos desarrollan tutorías de acompañamiento en busca de comprender la realidad y resaltar por medio de reflexiones, el sentido del desarrollo de la práctica; así mismo se utiliza como parámetro medir el aprendizaje de los aprendices con enfoque $u$ orientaciones en habilidades blandas y competencias organizacionales. La muestra está definida en tres modalidades: primera: generar ideas de negocio, segunda: planes de mejoramiento organizacional y tercera: contrato de aprendizaje. Finalizando su práctica los estudiantes exponen trabajos por medio de una muestra empresarial, donde los jurados mediante una lista de verificación califican: Análisis del trabajo elaborado de acuerdo a la modalidad escogida, presentación de ponencia y poster. En el análisis general de la muestra se encontró una relación significativa en el desarrollo de habilidades y la formación universitaria con su desempeño empresarial. Por otra parte, se evidenció que el tiempo establecido para acompañamiento del docente en la práctica profesional para el desarrollo de cada proyecto de los estudiantes, influye en la performance del trabajo. También se estima que a la hora de escoger el proyecto donde se debe realizar la práctica debe contarse con aprendizajes en las áreas delegadas por el empresario, para poder ofrecer un mejor desempeño futuro.

PALABRAS CLAVES: Prácticas profesionales, estudiantes, docentes, empresarios, formación universitaria.

\section{ABSTRACT:}

This article emphasizes the importance of the professional practice of Uniminuto university students during significant experiences in the business sector. The practices from their formative character, seek to consolidate as a reflexive and critical process helping to understand and interpret the reality of the profession in areas of knowledge with significant learning. They develop accompanying tutorials in search of understanding reality and highlighting, through reflections, the sense of development of the practice; Likewise, it is used as a parameter to measure the apprentices' learning with a focus or orientations in soft skills and organizational competencies. The sample is defined in three modalities: first: generate business ideas, second: organizational improvement plans and third: learning contract. At the end of their practice, the students present works through a business sample, where the jurors through a checklist qualify: Analysis of the work prepared according to the chosen modality, presentation of presentation and poster. In the general analysis of the sample a significant relationship was found in the development of skills and university training with their business performance. On the other hand, it was evidenced that the time established for the accompaniment of the teacher in the professional practice for the development of each student project, influences the performance of the work. It is also estimated that when choosing the project where the practice should be carried out, there should be learning in the areas delegated by the entrepreneur, in order to offer a better future performance.

KEYWORDS: Professional practices, students, teachers, entrepreneurs, university training.

\section{INTRODUCCIÓN}

Las prácticas profesionales forman parte de evidenciar la capacidad quetienen los estudiantes de enfrentar el mundo laboral, donde el alumno construye y demuestra sus habilidades blandas y saberes que han adquirido en tan solo ocho semestres de formación intelectual de su carrera profesional. El aprendiz puede adaptarse con facilidad, sujetarse a estilos diferentes de dirección, cumplir compromisos con entregas a tiempo y oportunas supervisadas.

Sin embargo, la poca experiencia profesional los hace confrontarse a punto de generar conciencia 
para prepararse y poseer mayores herramientas para enfrentarse de manera eficaz a un entorno empresarial, lo cual se convierte en un reto de superación personal. Las diversas condiciones de trabajo es un tema para analizar, la gran mayoría de las empresas prefieren no contar con estudiantes practicantes y prefieren pagar su cuota monetaria al gobierno, pero ese no es el caso para todas la empresas, son algunas en particular. En la actualidad, la situación económica del país hace que el empresario quiera contar con estudiantes practicantes, que no genere un compromiso económico, pero sí que realice funciones de trabajador. Las empresas que participaron en este ejercicio, comprendieron el significado de brindarle la oportunidad al estudiante para que efectuará su práctica profesional que le permitiera formarse como profesionista, teniendo en cuenta que no poseía beneficios de un trabajador, y en cambio otras no lo consideraron así.

Por otro lado, en una de las modalidades como la generación de ideas de negocio emprendimiento, se convirtió en una herramienta fundamental para algunos estudiantes que demostraron la creatividad, innovación, esfuerzo por consolidar su negocio demostrando gestión, habilidades blandas y competencias organizacionales, manifestando que se puede hacer empresa en nuestro país. En la modalidad contrato de aprendizaje, se notó la disposición y participación de empresas que contaban con el beneficio de incorporar estudiantes practicantes con contrato laboral a seis meses.

A través de la muestra empresarial denominada Ideochallenge, socialización de las prácticas profesionales exitosas, se pudo demostrar que a través de las prácticas profesionales se pueden proporcionar herramientas valiosas para el inicio del desempeño laboral de los estudiantes, para los futuros empresarios.

El objetivo de realizar este análisis es estudiar la relevancia de los saberes adquiridos durante la experiencia de la práctica, y que tan importante es la práctica profesional para los empresarios.

Dentro de los objetivos específicos los factores determinantes en el proceso de la práctica, habilidades blandas o conocimiento, ayuda a reconocer que tipo de aprendizaje está brindando el mundo laboral a los estudiantes, para así conocer la calidad de lo adquirido con lo proporcionado.

\section{LAS PRÁCTICAS PROFESIONALES EN EL MODELO PRAXOLÓGICO}

Según Juliao C. (2011). En el campo educativo existen diferentes modelos, en la corporación universitaria minuto de Dios Uniminuto, su eje fundamental del proceso de Enseñanza Aprendizaje en la Praxeología son Ver, Juzgar, Actuar y Devolver Colaborativamente. Donde por medio del Ver individual o grupal el docente, busca desarrollar y mantener en el profesional la actitud de indagar, y así mismo realice una reflexión crítica y consciente sobre su propia práctica surgiendo preguntas como ¿Qué estoy haciendo?; el juzgar, permite desenfocar la problemática de la práctica, donde se permite juzgar diversas teorías, de modo que pueda comprender la práctica y así mismo generar su propia teoría a través de la investigación, en el actuar, el estudiante construye en el tiempo y espacio la práctica, la gestión dirigida por tácticas guiadas a la acción, devolver colaborativamente es la representación, acción, devolver al entorno social, laboral, los conocimientos adquiridos en su proceso de formación en su quehacer profesional. Se puede destacar que este proceso de enseñanza fundamentado solo para la universidad minuto de Dios, proporciona un aprendizaje significativo, con el ánimo de que el estudiante no solo esté en clase magistral, sino que conozca y profundice en esta estrategia de enseñanza.

Para Juliao, C 2011. La Praxeología aparece como un esfuerzo de hermenéutica práctica 
que articula las funciones universitarias de investigación, de compromiso social crítico y de formación profesional al interior de un contexto publicitario. Por eso, ella sitúa plenamente el quehacer praxológico en un paradigma praxológico de investigación $\square$ acción formación en el que la práctica, en su contexto, es el punto de partida y de llegada, es generadora de teoría y de acción responsable. Justamente, la Praxeología no es sólo un ejercicio de investigación teórico o intelectual, sino y sobre todo, una práctica de responsabilidad y rendición de cuentas de los sujetos que la ejecutan. El modelo de la corporación Uniminuto, se enfoca en lo social donde las comunidades y población vulnerable son un objeto de estudio para realizar investigaciones y acciones que conlleven al beneficio de las este tipo de población. La Praxeología constituye una reflexión crítica sobre nuestro quehacer y la experiencia. Por eso la importancia del ejercicio de la práctica profesional es representativa en el campo laboral, ya que busca formar estudiantes socialmente responsables. Donde la razón Praxeologica es construir saberes a la luz del análisis de la práctica pensada, reflexionada, y no producto de la improvisación o del azar. Herrera, 2010.

\section{PRÁCTICA PROFESIONAL PENSADA, REFLEXIONADA, Y NO PRODUCTO DE LA IMPROVISACIÓN O DEL AZAR.}

El modelo praxeológico, propuesto por Juliao, fundamenta que los estudiantes se fortalezcan en aprendizajes significativos y socialmente pertinentes donde las practicas socio profesional, son procesos de generación donde existe la aplicación de conocimientos a partir de la experiencia, la realidad que ofrece el entorno laboral en el cual los estudiantes constantemente están; ellos son los constructores de una realidad social. Los practicantes Uniminuto generan un método de aproximación a la realidad que no pretende sólo observar para medir o valorar desde el investigador, sino para transformarlo y, desde su propia transformación, transforma sus prácticas y los contextos en los que interviene.

Aprender haciendo, experiencia que resalta los conocimientos de los practicantes donde demuestran a través de planes de mejoramiento organizacionales, emprendimiento y contratos de aprendizaje, la eficacia de lo aprendido a lo largo de su carrera, junto con sus habilidades blandas fraccionan la realidad socio profesional, generando un análisis del proyecto que llevaran a cabo, explicando la realidad del entorno laboral, comprendiendo las directrices de jefes y políticas de las empresas para lograr su objetivo y para finalizar actuando sobre lo establecido para llegar a un resultado final, donde esta realidad puede ser subjetiva.

Otros actores principales que participan en el proceso, son los docentes e interlocutores, quienes poseen el conocimiento para validar la práctica y encaminan al practicante a la reflexión redefiniendo el proyecto a lo largo del proceso. Encargados de formar seres humanos éticos, autónomos, consientes de sí mismos y de propiciar un tipo de educación social para la productividad.

Colombia necesita más profesionales éticos, preparados para ser responsables socialmente y competitivos laboralmente.

\section{PRÁCTICA PROFESIONAL COMO EFECTO ESPEJO}

Este ejercicio se llevó a cabo como efecto espejo, $\square \square$ comprobar lo que estamos intentando hacer". Los 35 estudiantes de noveno semestre, llevaron a cabo planes de mejoramiento organizacional, emprendimiento y algunos contratos de aprendizaje; donde desarrollaron actividades concretas con las empresas y comunidades con quienes interactuaron durante el proceso de lo programado, las empresas que participaron en el proceso fueron: Editorial 
Norma, Policía Nacional, Gems Ingeniería, Eforsalud, Avidanti SAS, Petrosantander, Universidad Santo Tomas, Distribuidora Rayco SAS, Metrogas de Colombia, SA, Motocentro SAS, Quimicur, Sena, Cajasan, Vifenalco Ltda, Banco Colpatria, Postobon SA, Nacional de Papeles, Uniminuto, Frimac SA, Transporte Líquidos de Colombia SA, Grupo Motor SAS, Agencia Logística de las Fuerzas Militares de Colombia, Eme Ingeniería SA, experiencia que permitió identificar la problemática en cada sitio de trabajo, donde los practicantes pusieron toda su energía, amor y pasión a lo planteado por las directivas y docente en realizar.

En primera instancia los estudiantes ejecutaron una observación (VER) de acuerdo a las vivencias e implicaciones para hacer un diagnóstico inicial de su proyecto, y sigue luego con las reflexiones, críticas e interpretación (JUZGAR), donde aparecen una serie de circunstancias esperadas y no esperadas, entonces, se busca identificar de manera rápida lo oculto para generar la propuesta de acción (ACTUAR), llevándolos al proceso de evaluación y reflexión (DEVOLUCIÓN CREATIVA), produciendo la transformación empresarial y la satisfacción profesional de aplicar los conocimientos adquiridos en la formación profesional, siendo esta una carta de presentación como experiencia ante el mundo laboral. Esta devolución creativa fue expuesta en el evento académico denominado "Idea challenge socialización de las prácticas profesionales exitosas Administración de Empresas $\square \square$, donde por medio de una muestra empresarial se contó con la participación de empresarios, invitados, que pudieron apreciar las experiencias vividas y reflejadas por los practicantes a través de ponencias y poster. Este ejercicio profesional contó con la evaluación de docentes y estudiantes de todos los semestres del programa.

\section{ABORDAJE METODOLÓGICO IDEA CHALLENGE SOCIALIZACIÓN DE PRÁCTICAS PROFESIONALES EXITOSAS ADMINISTRACIÓN DE EMPRESAS UNIMINUTO.}

Se aplicó, un cuestionario sobre percepción, expectativas y conocimiento del proyecto de la práctica profesional a 93 estudiantes y 10 docentes, de edades comprendidas entre 18 a 50 años, los cuales corresponden a la carrera de Administración de Empresas. Los estudiantes pertenecen a los semestres del 1 a 10 de la corporación universitaria minuto de Dios Uniminuto; la aplicación se hizo en una sola etapa; el $66 \%$ son mujeres y el $34 \%$ hombres.

El cuestionario está compuesto por 5 preguntas, divida en tres partes, la primera comprende dos preguntas, a través de los cuales se solicitó a los estudiantes conocer sus opiniones sobre la práctica, el objetivo que persigue el estudiante con su proyecto, la expectativa que genera y las condiciones en que realizaron su práctica profesional. La valoración sobre estas preguntas conductuales y funcionales determina las habilidades blandas, capacidad de resolución de conflictos y desempeño laboral para realizar sus actividades, ayudando al programa a analizar como las prácticas han contribuido en el desarrollo personal y profesional del profesionista.

La segunda relaciona aspectos para mejorar los procesos del proceso e implementar mejoras en el trabajo desarrollado en las instituciones. La valoración sobre las situaciones que deben manifestarse se evaluó por medio de la escala de Likert, donde se analizaron los datos revisando las perspectivas de cada una de las dimensiones de la pregunta y sobre todo las condiciones de las prácticas profesionales.

Finalizando, la tercera parte comprende las sugerencias del evaluador en cuanto a la gestión 
de la muestra empresarial, invitados, ponencias, posters.

\section{ANÁLISIS DE LOS RESULTADOS}

El análisis de las respuestas de los 93 alumnos y 10 docentes de Administración de Empresas que llevaron a cabo las evaluaciones en el evento académico Idea challenge en el presente año. Donde fue posible a través de este ejercicio profesional, desarrollar reflexiones vinculadas al adelanto de las prácticas profesionales.
1. La primera pregunta hace referencia al objetivo que persigue el estudiante al realizar su práctica profesional de los cuales se destacan aspectos como: analizar su entorno, generar su idea de proyecto, accionar para llevarla a cabo y gestionar o devolver colaborativamente aplicando sus conocimientos con ética profesional. (G1). Según el gráfico se puede apreciar que el objetivo de gran importancia es adquirir la experiencia para facilitar su proceso en el campo laboral, seguido de conocer cómo funciona realmente una organización.

Gráfica 1. Objetivos de los estudiantes al realizar la práctica profesional

\section{G.1. Objetivo de los Estudiantes al realizar la Práctica Profesional}

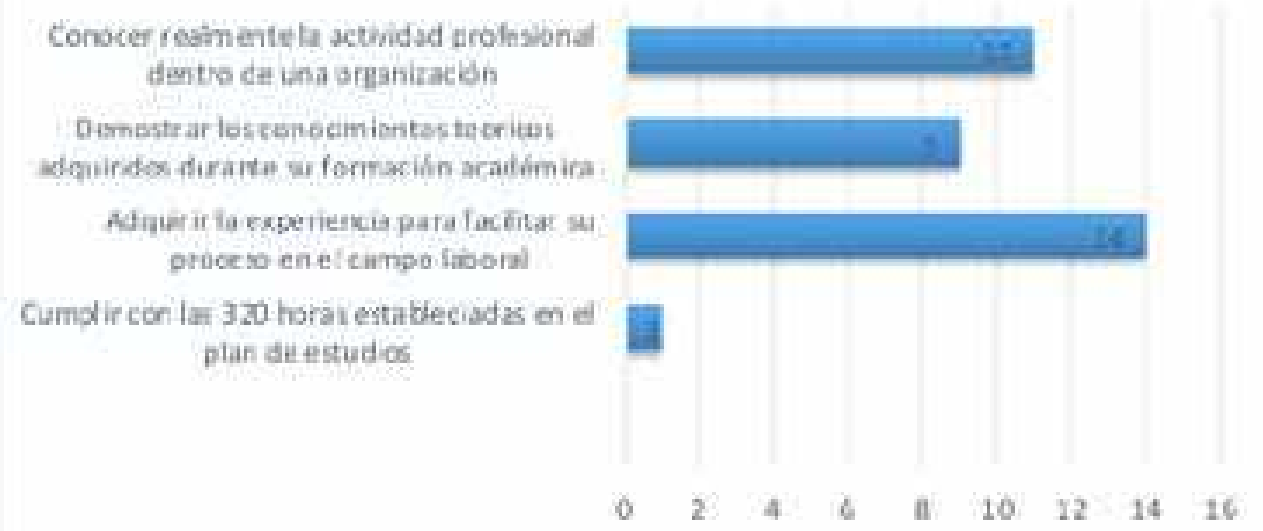

Fuente: Repositorio - Programa de formación Administración de Empresas Uniminuto Bucaramanga

2. La segunda pregunta menciona la expectativa o impacto que generó el estudiante en su práctica profesional de acuerdo al proyecto realizado (G2). 
Gráfica 2. Qué generó más impacto de los proyectos observados

\section{G.2. Qué generó más impacto de los proyectos observados}

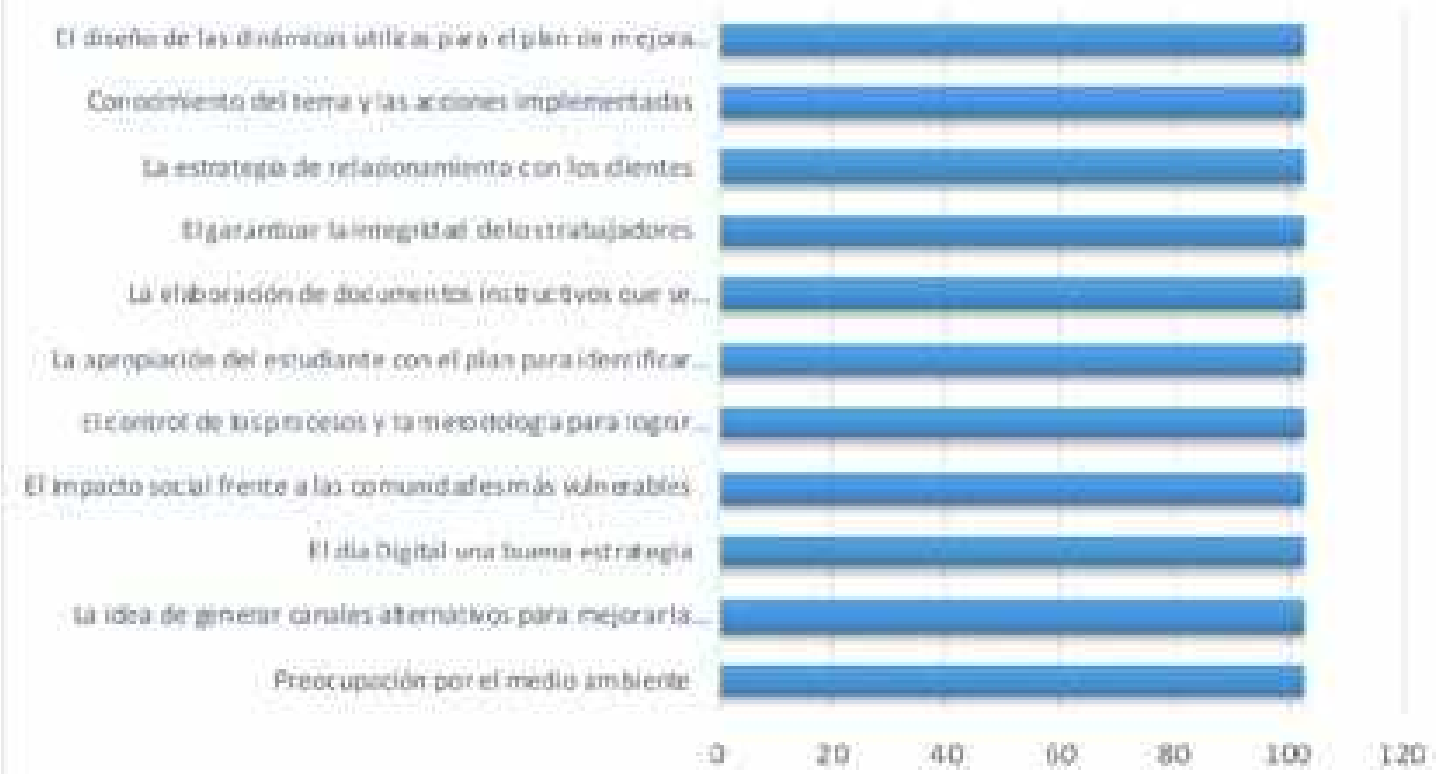

Fuente: Repositorio - Programa de formación Administración de Empresas Uniminuto Bucaramanga

Se puede observar que en igual grado de importancia se encontraron: Preocupación por el medio ambiente, la idea de generar canales alternativos para mejorar la atención a los clientes, el día Digital una buena estrategia, el impacto social frente a las comunidades más vulnerables, el control de los procesos y la metodología para lograr este análisis, la apropiación del estudiante con el plan para identificar el problema, la elaboración de documentos instructivos que se convirtieron útiles para la empresa, el garantizar la integridad de los trabajadores, la estrategia de relacionamiento con los clientes, conocimiento del tema y las acciones implementadas, el diseño de las dinámicas utilizas para el plan de mejora en la empresa; estas son herramientas útiles que se pueden aplicar en cualquier organización.

3. La tercera pregunta, hace referencia a identificar en los proyectos propuestos por los practicantes, cuales son los aspectos más relevantes para implementar las mejoras en el trabajo observado y la respuesta de los evaluadores se pueden apreciar en la gráfica (G3). 
Gráfica 3. Qué aspectos considera usted puede aportar a mejorar los procesos

\section{Qué aspectos considera usted puede aportar para mejorar los procesos o implementar mejoras en el trabajo observado}

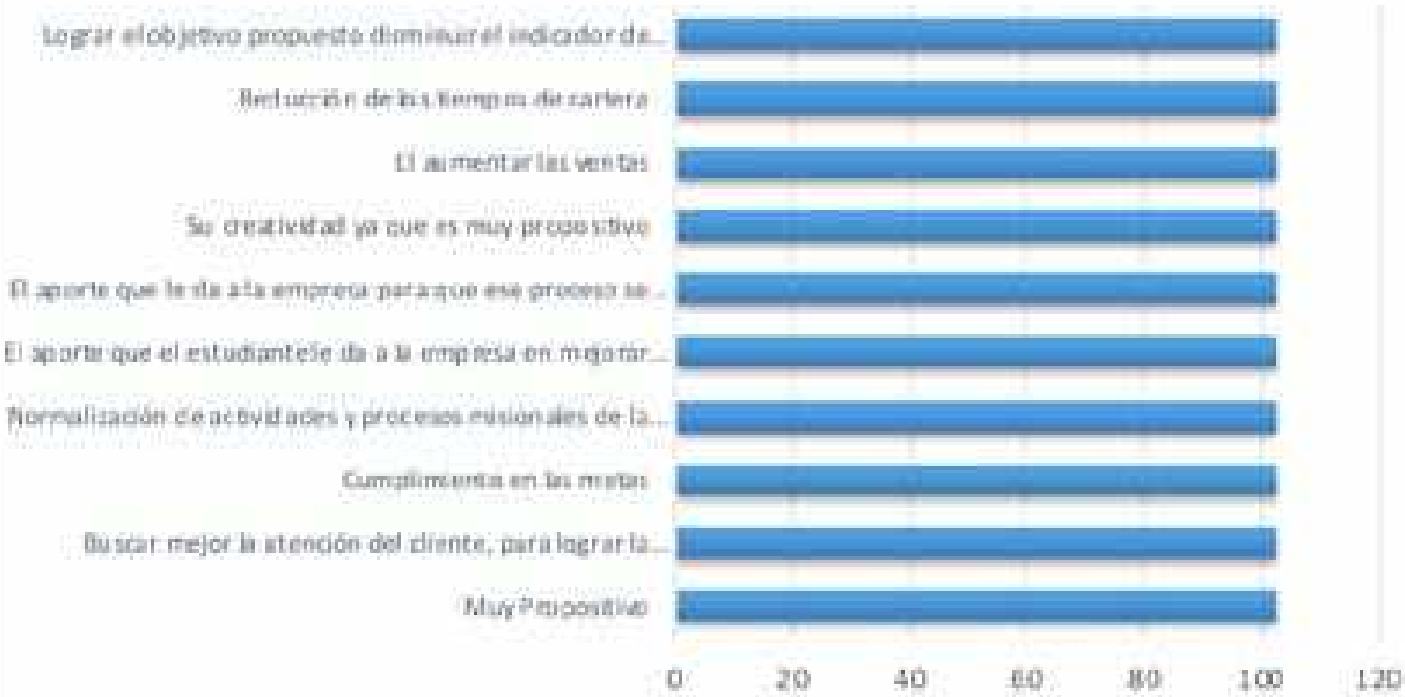

Fuente: Repositorio - Programa de formación Administración de Empresas Uniminuto Bucaramanga

Las características más relevantes que los jóvenes consideraron es la búsqueda del cumplimiento en metas, mejorar la calidad con los clientes y lograr su fidelización, mejorar los procesos de calidad en las instituciones, la importancia de los aportes de los practicantes a la empresa en el área asignada, se puedan implementar en las demás, la creatividad es muy propositiva, el aumento de las ventas, la disminución de cartera, objetivos que se pueden llegar a lograr; pero hay que tener en cuenta que cada proyecto expuesto ha sido desarrollado en un semestre.

4. En la cuarta pregunta se le brinda la oportunidad de que los estudiantes y docentes puedan calificar los proyectos expuestos por los practicantes. (G4). 
Gráfica 4. Proporcione la calificación que usted considera que se merece los proyectos.

\section{Proporcione la calificación que usted considera que se merece los proyectos}
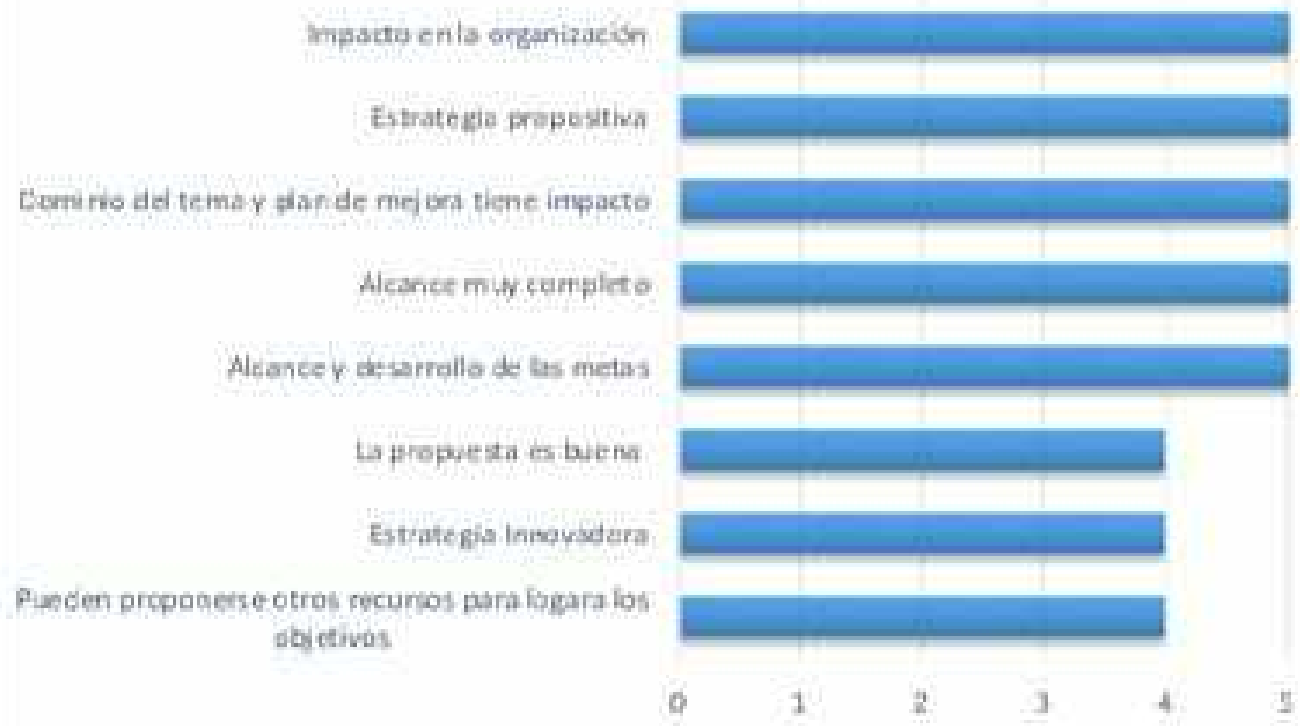

Fuente: Repositorio - Programa de formación Administración de Empresas Uniminuto Bucaramanga

Como lo demuestra la gráfica G.4, donde el sentido demostrado en las prácticas profesionales por parte de los estudiantes, es estructurado desde los objetivos y cumple con las expectativas de los estudiantes. Finalmente, una pregunta abierta que proporciona reflexiones finales de las prácticas profesionales para los estudiantes de noveno semestre y programa.

- Mas disponibilidad de tiempo de los invitados

- $\quad$ Convenios empresariales

- Buen ejercicio para el mejoramiento académica

- Mayor disponibilidad de tiempo en la elaboración del proyecto

- Involucrar más empresarios, para futuras practicas

- $\quad$ Ampliar el campo de investigación
- Más continuidad de las ferias empresariales

- $\quad$ Buen conocimiento del proyecto

- Innovar en proyectos

- Más tiempo en el desarrollo de las practicas

- $\quad$ Ayuda financiera para los estudiantes

- Mayor conocimiento en los procesos de la empresa

\section{CONCLUSIONES}

La práctica profesional es una estrategia de proyección social, que se concibe como una actividad pedagógica complementaria a la formación delestudiantedesdesuárea disciplinar, adquiriendo importancia en la sociedad y el mundo laboral. Las prácticas profesionales 
permiten al practicante transformar situaciones determinadas, integrar la teoría y la práctica, desarrollar las competencias profesionales, incorporando sus saberes, que se inserte en el mundo laboral creando oportunidades futuras, que realice un ejercicio de reflexión sobre su propia práctica fortaleciendo sus conocimientos y sentido de adaptación, que el estudiante se permita constituirse en un espacio de aprendizaje significativo aplicando la ética profesional y servicio, ya que las prácticas profesionales en Uniminuto están orientadas a desarrollar estudiantes socialmente responsables y con habilidades blandas para dar solución a diversas problemáticas.

Se puede resaltar que las tutorías de acompañamiento orientadas por la docente (virtualesypresenciales) siendoestasunaguía de aprendizaje, para cada estudiante, proporcionó en el practicante: La reflexión sobre la práctica, el trabajo sobre los elementos conceptuales, metodológicos y pedagógicos que fortalecen al estudiante en el desarrollo de la práctica y que desde luego nutren su conocimiento disciplinar, el intercambio y la socialización de experiencias a partir de la realización de las mismas, la planeación y sistematización de las prácticas, la aclaración de dudas que surjan durante el proceso formativo, la orientación disciplinar del campo de práctica, obteniendo como resultado estudiantes líderes, capaces, emprendedores, dispuestos a aceptar el reto de enfrentar una práctica con profesionalismo, dedicación, pasión y compromiso social - empresarial, demostrando cada uno su talento.

Este tipo de actividades académicas como el Idea challenge, se necesitan, ya que su propósito radica en transmitir el conocimiento de los estudiantes y se enriquezca su formación integral, así mismo brindar la oportunidad a los otros estudiantes de los semestres inferiores de preparar sus expectativas frente a su futura práctica profesional.

\section{REFERENCIAS BIBLIOGRÁFICAS:}

Andreozzi, M. (2011). Las prácticas profesionales de formación como experiencias de pasaje $y$ tránsito identitario. Archivos de Ciencias de la Educación, 4a. época, 5(5).

Blander, R. (1997). Use su cabeza para variar: Sub-modalidades en programación Neurol Lingüística. Editorial Cuatro vientos, Santiago de Chile.

Grinder, J. (1998), Blander, R. (1998). La estructura de la Magia (V1): Lenguaje y Terapia. Editorial Cuatro vientos, Santiago de Chile.

Deming, W. Medina, J. (1989). Calidad, productividad y competitividad: la salida de la crisis. Edición Díaz de Santos S.A, Madrid.

Juliao, C (2011) El enfoque Praxeológico. Editor Corporación Universitaria Minuto de Dios. 